\title{
AVALIAÇÃO DA QUALIDADE DOS REGISTROS DE ENFERMAGEM EM UNIDADES DE TERAPIA INTENSIVA: REVISÃO INTEGRATIVA
}

\section{ASSESSMENT OF THE QUALITY OF NURSING RECORDS IN INTENSIVE THERAPY UNITS: INTEGRATION REVIEW}

\author{
${ }^{1}$ Hiasmin Batista Rodrigues \\ ${ }^{2}$ Fabiene Lima Parente
}

${ }^{1}$ Enfermeira na Atenção Básica à Saúde de Uruoca (CE). Especialista em Auditoria e Gestão em Serviços de Saúde pela Faculdade Padre Dourado - FACPED. hiasmin.6@gmail.com. Rua Maestro Pedro Peixoto, no 90, 62460-000, Centro, Uruoca (CE)

2Professora Mestre em Ensino na Saúde pela Universidade Estadual do Ceará UECE, especialista em Gestão em Serviços de Saúde e Auditoria pelo Instituto Executivo de Formação - IEF. Coordenadora Hospitalar do Hospital do Coração de Sobral e professora de pós-graduação no Instituto Executivo de Formação, nas Faculdades INTA, no Instituto Lato Sensu e na Escola de Formação em Saúde da Família Visconde de Saboia. 


\section{RESUMO}

INTRODUÇÃO: Esta revisão objetiva avaliar a qualidade dos registros dos cuidados de enfermagem prestados aos pacientes em estado críticos, a partir da análise das publicações científicas sobre a importância dos registros e identificar fragilidades e estratégias para a melhoria da qualidade dos registros. METODOLOGIA: Trata-se de uma revisão integrativa, realizada em abril de 2017, norteada pela pergunta: Qual a qualidade dos registros de enfermagem no cenário de cuidados críticos? Com busca na Biblioteca Virtual de Saúde, com artigos completos disponíveis, em português, com publicação nos últimos cinco anos e como resultado da permuta de descritores que englobam a temática. RESULTADOS E DISCUSSÕES: Diante dos artigos encontrados, as temáticas relatavam a carência de registros, incompletudes, falhas e escassez, além da explanação sobre estratégias para resolutividade das falhas encontradas. Através dos registros foi concretizada a formulação situacional da assistência, onde informações eram extraídas para diagnóstico e características dos serviços, identificando a necessidade de práticas para reestruturar a assistência, onde foi evidenciado que formulários manuais e eletrônicos, podem ser utilizados para a implementação do processo de enfermagem, pois dispõem das funções necessárias a sua aplicação na forma correta. CONCLUSÃO: Há a necessidade de registros completos e claros, como forma de continuidade do cuidado, e fato simples de ser executado e compreendido pelos profissionais.

Palavras-chaves: Registros de enfermagem; Avaliação em saúde; UTI

\section{ABSTRAT}

INTRODUCTION: This review aims to evaluate the quality of nursing care records provided to critically ill patients, based on the analysis of scientific publications on the importance of registries and identify weaknesses and strategies for improving the quality of records. METHODOLOGY: This is an integrative review, conducted in April 
2017, guided by the question: What is the quality of nursing records in the critical care setting? With search in the Virtual Health Library, with complete articles available in Portuguese, with publication in the last five years and as a result of the exchange of descriptors that encompass the theme. RESULTS AND DISCUSSIONS: In the face of the articles found, the subjects reported the lack of records, incompleteness, failure and scarcity, as well as an explanation of strategies for solving the flaws found. Through the records, the situational formulation of the assistance was materialized, where information was extracted for the diagnosis and characteristics of the services, identifying the need for practices to restructure assistance, where it was evidenced that manual and electronic forms can be used for the implementation of the nursing process, since they have the necessary functions to apply it in the correct form. CONCLUSION: There is a need for complete and clear records, as a form of continuity of care, and simple fact to be executed and understood by professionals.

Keywords: Nursing records; Health evaluation; Intensive care units.

\section{INTRODUÇÃO}

A qualidade assistencial é considerada um processo complexo, no qual, permite identificar e consolidar os aspectos vulneráveis na dinâmica de trabalho da equipe de enfermagem e independentemente dos programas avaliativos adotados considera-se importante o compromisso assumido com a qualidade da assistência do cuidado'1.

Como forma de subsídio à qualidade assistencial ofertada, os registros da equipe de enfermagem trazem informações que permitem um julgamento clínico objetivo diante das informações descritas, pois estas reverberam o atendimento e tratamento prestado ${ }^{2}$, devido serem os profissionais que prestam auxilio intenso no período de internação.

A Sistematização da Assistência de Enfermagem é considerada o principal instrumento para o desempenho sistemático da prática profissional, elevando a 
qualidade assistencial e beneficiando todos os sujeitos envolvidos, então, os registros se tornaram um instrumento ético e legal, que respalda as ações do cuidado $^{3}$, e ainda, perfazem uma intercomunicação permanente entre os membros da equipe multiprofissional ${ }^{2}$, posto que, o sub-registro implica em não realização de ações principalmente nos cenários críticos de um hospital, onde os mínimos detalhes da assistência necessitam ser conhecidos por todos da equipe, reforçando o sistema comunicativo, onde, por vezes, tem se a falha na comunicação oral.

Desse modo, registros completos, possuem grande relevância comunicativa nos setores, sendo que na unidade de terapia intensiva esta necessidade se torna elevada, visto que é o local onde se encontra a maior concentração de pacientes críticos, intensificando sua importância dentro da Sistematização da Assistência de Enfermagem (SAE) possibilitando a verificação dos procedimentos realizados e coordenação dos cuidados prestados, facilitando o replanejamento do plano assistencial, estabelecendo um padrão para a qualidade do cuidado 4 .

Assim, em um setor com características peculiares, que reúne um montante de pessoas em estado crítico, há a necessidade de uma avaliação dos registros efetivados para que demandem perspectivas aos gestores da unidade, concernentes às práticas ofertadas.

Assim posto, esta revisão contribuirá para reorientação das práticas e subsequente qualificação da atenção à saúde da equipe de enfermagem no cuidado a pacientes em unidades de terapia intensiva, pois objetiva avaliar a qualidade dos registros de cuidados de enfermagem prestados aos pacientes em estado crítico através de uma revisão integrativa, a partir da análise das publicações cientificas sobre a importância dos registros e identificando suas fragilidades e estratégias que visam a melhoria da qualidade dos registros.

\section{FUNDAMENTAÇÃO TEORICA}

A auditoria dentro da saúde foi introduzida em meados do século $X X$, sendo utilizada como um instrumento de análise da qualidade da assistência, por intermédio dos registros nos prontuários dos pacientes ${ }^{5}$. Consistindo ainda, na avaliação sistemática e formal de uma atividade para determinar se ela está sendo 
realizada de acordo com o seu devido objetivo, destarte, o principal propósito da auditoria de enfermagem é apontar fragilidades nos serviços para o fornecimento de dados concretos para que decisões sejam tomadas ${ }^{6}$.

A avaliação da qualidade pode ocorrer através das três dimensões propostas por Donabedian: estrutura, processo e resultado, a fim de complementarem-se para a obtenção de melhores resultados. No entanto para identificação mais específica das divergências, por vezes avalia-se separadamente cada etapa. A estrutura são os recursos físicos, humanos, materiais, equipamentos e financeiros necessários para a assistência à saúde; $O$ processo, se refere às atividades envolvendo profissionais de saúde e usuários, inclui diagnóstico, tratamento, aspectos éticos de relação profissional, equipe de saúde e paciente; e o resultado, que corresponde ao produto final da assistência prestada, considerando a saúde, satisfação de padrões e expectativas dos usuários ${ }^{7}$. Para fins do presente artigo, foi avaliada a dimensão processo através das ações de registro pela equipe de enfermagem, uma vez que sua importância é evidenciada na continuidade do cuidado, de forma prática e contundente.

A auditoria em enfermagem possibilita avaliar sistematicamente a qualidade da assistência através das anotações de enfermagem nos prontuários dos pacientes, além do fato de identificar as inconsistências contidas nestes. Os registros facilitam a continuidade da assistência prestada, quando o ato de registrar é escasso ou incompleto compromete não só a equipe de enfermagem, mas também a qualidade assistencial ${ }^{8}$.

Os profissionais de enfermagem são responsáveis por registrar nos prontuários dos pacientes, as informações inerentes ao processo de cuidar, garantindo a continuidade e a qualidade da assistência ${ }^{9}$. A avaliação dos registros de enfermagem é uma ação importante, pois permite verificar não somente a qualidade de seu conteúdo, mas também a execução do exercício profissional da equipe ${ }^{10}$, destarte, a avaliação de enfermagem e o seu registro em todos os contextos do cuidado, principalmente em terapia intensiva, são de extrema importância para reconhecimento das carências do ser, influenciando na tomada de decisões clínicas e na execução de intervenções ${ }^{11}$. 


\section{METODOLOGIA}

Trata-se de uma pesquisa do tipo revisão integrativa, com abordagem qualitativa, com coleta de dados realizada no mês de abril 2017. Este método de pesquisa é importante, pois possibilita a compilação dos estudos publicados e permite estruturar conclusões gerais sobre um determinado assunto ${ }^{12}$.

Foram percorridas as seis etapas sugeridas por Mendes $^{13}$, para construção da revisão integrativa: A primeira etapa foi identificar a temática através da elaboração da pergunta norteadora da pesquisa que foi: Qual a qualidade dos registros de enfermagem no cenário de cuidados críticos? A segunda etapa engloba os critérios de inclusão da pesquisa, quais sejam: Resultados de busca encontrados na Biblioteca Virtual de Saúde (BVS), artigos com texto completo disponíveis e em português, além de sua publicação ter ocorrido nos últimos cinco anos, e sendo resultado da permutação entre os descritores Avaliação em Saúde and UTI and Registros de Enfermagem, Avaliação em Saúde and UTI, Avaliação em Saúde and Registros de Enfermagem com caracterização de seus assuntos principais serem: Qualidade da assistência à saúde, unidades de terapia intensiva e indicadores da qualidade da assistência. Com a escolha dos descritores sendo por suas definições abraçarem a pergunta norteadora do estudo. Os critérios de exclusão foram: artigos repetidos e com assunto avaliado a partir do título divergente do objeto de estudo.

$\mathrm{Na}$ terceira etapa, ocorreu a seleção dos trabalhos científicos, de acordo com os critérios de inclusão e exclusão, com posterior formulação de categorias a partir da repetição de temáticas. Na quarta etapa tem-se a avaliação dos achados nos artigos, e com efetiva interpretação destes achados sugerida pela quinta etapa. E como última etapa teve-se a síntese dos resultados encontrados nos artigos.

Assim, após os critérios de inclusão e exclusão foram encontrados os seguintes artigos:

Quadro 1 - Resultados da busca de artigos de acordo com a estratificação de critérios de inclusão e exclusão, 2017. 
Artigo Original

Atenção à saúde

\begin{tabular}{|c|c|c|c|c|c|}
\hline $\begin{array}{l}\text { Critérios } \\
\text { de } \\
\text { busca }\end{array}$ & Descritores & $\begin{array}{l}\text { Avaliação } \\
\text { em Saúde } \\
\text { and UTI }\end{array}$ & $\begin{array}{l}\text { Avaliação } \\
\text { em Saúde } \\
\text { and } \\
\text { Registros de } \\
\text { Enfermagem }\end{array}$ & $\begin{array}{l}\text { Registros de } \\
\text { Enfermagem } \\
\text { and UTI }\end{array}$ & $\begin{array}{l}\text { Avaliação } \\
\text { em Saúde } \\
\text { and UTI and } \\
\text { Registros de } \\
\text { Enfermagem }\end{array}$ \\
\hline \multicolumn{2}{|c|}{ Artigos sem Critérios } & 3236 & 1929 & 386 & 56 \\
\hline \multicolumn{2}{|c|}{$\begin{array}{l}\text { Artigos: } \\
\text { - Com texto completo } \\
\text { disponível } \\
\text { - Em Português } \\
\text { - Publicação nos } \\
\text { últimos cinco anos }\end{array}$} & 86 & 68 & 34 & 11 \\
\hline \multicolumn{2}{|c|}{$\begin{array}{l}\text { Assuntos Principais: } \\
\text { - Qualidade da } \\
\text { assistência à saúde } \\
\text { - Unidades de Terapia } \\
\text { Intensiva } \\
\text { - Indicadores de } \\
\text { qualidade da } \\
\text { assistência }\end{array}$} & 44 & 12 & 20 & 8 \\
\hline \multicolumn{2}{|c|}{$\begin{array}{l}\text { Artigos com a temática } \\
\text { convergente ao } \\
\text { objetivo do estudo }\end{array}$} & 5 & 3 & 4 & 3 \\
\hline \multicolumn{2}{|c|}{ Artigos Repetidos } & 3 & 2 & 2 & 2 \\
\hline \multicolumn{2}{|c|}{ Total da Amostra } & 2 & 1 & 2 & 1 \\
\hline
\end{tabular}

Fonte: Elaborado pelos autores, 2017.

A partir disto, foi construída uma tabela com os artigos encontrados onde se descreve o título, o assunto, o periódico, o ano de publicação e finalmente discutido suas principais concordâncias e discordâncias.

\section{RESULTADOS E DISCUSSÕES}


Dos quinze artigos que atendiam a todos os critérios, nove deles repetiam-se dentro de cada permutação dos descritores, sendo estes excluídos totalizando um quantitativo de seis artigos para análise final. Todos os periódicos e cenários dos estudos analisados estavam localizados no centro-sul do país.

Diante dos artigos encontrados, as abordagens temáticas abrangiam principalmente a carência de registros, bem como sua incompletude, deixando os registros falhos e escassos, além da explanação sobre as estratégias que visam contornar as falhas encontradas.

Quadro 2. Apresentação dos artigos segundo identificação, assunto, contribuições do estudo, 2017.

\begin{tabular}{|c|c|c|c|c|}
\hline Número & Titulo & Periódico/Ano & Assunto & $\begin{array}{l}\text { Contribuições do } \\
\text { Estudo }\end{array}$ \\
\hline 1 & $\begin{array}{l}\text { Registro da } \\
\text { Avaliação de } \\
\text { Enfermagem } \\
\text { em Terapia } \\
\text { Intensiva: } \\
\text { Discurso do } \\
\text { sujeito } \\
\text { coletivo }^{11}\end{array}$ & $\begin{array}{l}\text { Revista de } \\
\text { Enfermagem da } \\
\text { UFSM/2015 }\end{array}$ & $\begin{array}{l}\text { Compreender } \\
\text { as } \\
\text { percepções } \\
\text { de } \\
\text { enfermeiros } \\
\text { sobre a } \\
\text { avaliação de } \\
\text { enfermagem } \\
\text { e seu } \\
\text { respectivo } \\
\text { registro na } \\
\text { terapia } \\
\text { intensiva }\end{array}$ & $\begin{array}{l}\text { Apresenta a } \\
\text { praticidade e rapidez } \\
\text { para a efetivação dos } \\
\text { registros no processo } \\
\text { de enfermagem, no } \\
\text { entanto, foi identificado } \\
\text { a valoração de } \\
\text { aspectos físicos em } \\
\text { detrimento dos } \\
\text { psicossociais, sendo } \\
\text { observado a } \\
\text { necessidade de } \\
\text { aperfeiçoamento da } \\
\text { equipe no processo de } \\
\text { registrar e atualização } \\
\text { do instrumento para } \\
\text { extração de }\end{array}$ \\
\hline
\end{tabular}




\begin{tabular}{|c|c|c|c|c|}
\hline & & & & informações \\
\hline 2 & $\begin{array}{l}\text { Processo de } \\
\text { Enfermagem: } \\
\text { comparação } \\
\text { do registro } \\
\text { manual } \\
\text { versus } \\
\text { eletrônico }^{14}\end{array}$ & $\begin{array}{l}\text { Journal of } \\
\text { Health } \\
\text { Informatics/2015 }\end{array}$ & $\begin{array}{l}\text { Verificar se a } \\
\text { implantação } \\
\text { de um } \\
\text { software para } \\
\text { registros de } \\
\text { anotações } \\
\text { atribuiu } \\
\text { ganhos na } \\
\text { assistência }\end{array}$ & $\begin{array}{l}\text { Identificou-se a } \\
\text { utilização de um } \\
\text { instrumento de registro } \\
\text { eletrônico frente ao } \\
\text { registro manual, } \\
\text { permitindo uma maior } \\
\text { compreensão sobre o } \\
\text { processo de } \\
\text { enfermagem aplicado e } \\
\text { sobre o suporte à } \\
\text { assistência, apesar de } \\
\text { o tempo mesurado em } \\
\text { ambos os registros ter } \\
\text { sido equivalente }\end{array}$ \\
\hline
\end{tabular}

Continua

\begin{tabular}{|c|c|c|c|c|}
\hline Número & Titulo & Periódico/Ano & Assunto & $\begin{array}{l}\text { Contribuições do } \\
\text { Estudo }\end{array}$ \\
\hline 3 & $\begin{array}{l}\text { Qualidade da } \\
\text { Assistência } \\
\text { de } \\
\text { Enfermagem } \\
\text { em Unidade } \\
\text { de Terapia } \\
\text { Intensiva de } \\
\text { um hospital } \\
\text { Escola }{ }^{1}\end{array}$ & $\begin{array}{l}\text { Revista Gaúcha } \\
\text { de } \\
\text { Enfermagem/2013 }\end{array}$ & $\begin{array}{l}\text { Avaliar a } \\
\text { qualidade } \\
\text { da } \\
\text { assistência } \\
\text { de } \\
\text { enfermagem } \\
\text { ofertada em } \\
\text { terapia } \\
\text { intensiva }\end{array}$ & $\begin{array}{l}\text { Diante da avaliação } \\
\text { através de um } \\
\text { checklist, a } \\
\text { qualidade do serviço } \\
\text { prestado foi } \\
\text { classificada como } \\
\text { sofrível, } \\
\text { estabelecendo um } \\
\text { diagnóstico } \\
\text { situacional das } \\
\text { práticas de }\end{array}$ \\
\hline
\end{tabular}

Revista Inova Saúde, Criciúma, vol.9, n. 1, jul. 2019. 


\begin{tabular}{|c|c|c|c|c|}
\hline & & & & enfermagem. \\
\hline 4 & $\begin{array}{l}\text { Avaliação da } \\
\text { qualidade } \\
\text { das } \\
\text { anotações de } \\
\text { enfermagem } \\
\text { em unidade } \\
\text { semi- } \\
\text { intensiva }^{2}\end{array}$ & $\begin{array}{l}\text { Escola Anna Nery } \\
\text { (impresso)/2012 }\end{array}$ & $\begin{array}{l}\text { Avaliar a } \\
\text { qualidade } \\
\text { das } \\
\text { anotações } \\
\text { realizadas } \\
\text { em unidade } \\
\text { semi- } \\
\text { intensiva }\end{array}$ & $\begin{array}{l}\text { Frente a } \\
\text { necessidade de } \\
\text { anotações completas } \\
\text { e precisas, neste } \\
\text { estudo, em nenhum } \\
\text { dos critérios de } \\
\text { preenchimento dos } \\
\text { registros alcançou } \\
\text { um bom padrão de } \\
\text { qualidade, } \\
\text { apresentando } \\
\text { ausência de } \\
\text { registros sobre } \\
\text { necessidades dos } \\
\text { pacientes. }\end{array}$ \\
\hline 5 & $\begin{array}{l}\text { Valoração e } \\
\text { registros } \\
\text { sobre higiene } \\
\text { oral de } \\
\text { pacientes } \\
\text { intubados } \\
\text { nas unidades } \\
\text { de terapia } \\
\text { intensiva }{ }^{15}\end{array}$ & $\begin{array}{l}\text { Revista Mineira } \\
\text { de } \\
\text { Enfermagem/2016 }\end{array}$ & $\begin{array}{l}\text { Mensurar a } \\
\text { valoração } \\
\text { da higiene } \\
\text { bucal e } \\
\text { verificar se } \\
\text { há registros } \\
\text { e } \\
\text { prescrições } \\
\text { da equipe } \\
\text { de } \\
\text { enfermagem } \\
\text { sobre a } \\
\text { higiene oral } \\
\text { dos } \\
\text { pacientes }\end{array}$ & $\begin{array}{l}\text { Apesar do } \\
\text { reconhecimento } \\
\text { sobre a importância } \\
\text { de procedimentos } \\
\text { relacionados a } \\
\text { higiene bucal, o } \\
\text { registro destes não } \\
\text { era pertinentemente } \\
\text { realizado, bem como } \\
\text { identificado a } \\
\text { ausência de } \\
\text { diagnósticos de } \\
\text { enfermagem para } \\
\text { este fim. }\end{array}$ \\
\hline
\end{tabular}




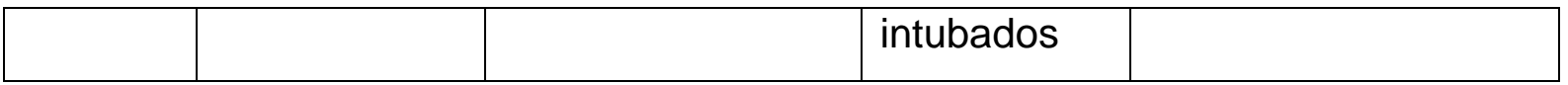

Continua

\begin{tabular}{|c|c|c|c|c|}
\hline Número & Titulo & Periódico/Ano & Assunto & $\begin{array}{l}\text { Contribuições do } \\
\text { Estudo }\end{array}$ \\
\hline 6 & $\begin{array}{l}\text { Mensuração } \\
\text { do tempo dos } \\
\text { registros } \\
\text { manual e } \\
\text { eletrônico da } \\
\text { Sistematização } \\
\text { da Assistência } \\
\text { de } \\
\text { Enfermagem } \\
\text { em Unidade de } \\
\text { Terapia } \\
\text { Intensiva }{ }^{16}\end{array}$ & $\begin{array}{l}\text { Jounal of Health } \\
\text { Informatics/2012 }\end{array}$ & $\begin{array}{l}\text { Mensurar o } \\
\text { tempo que } \\
\text { enfermeiros } \\
\text { utilizam para } \\
\text { os registros } \\
\text { assistenciais } \\
\text { de forma } \\
\text { manual e } \\
\text { eletrônica }\end{array}$ & $\begin{array}{l}\text { Foi observado que é } \\
\text { gasto mais tempo com } \\
\text { o registro eletrônico } \\
\text { das tarefas da } \\
\text { Sistematização de } \\
\text { Enfermagem, no } \\
\text { entanto, estes } \\
\text { registros são mais } \\
\text { detalhados e possuem } \\
\text { informações mais } \\
\text { complexas do exame } \\
\text { físico em comparação } \\
\text { com os achados } \\
\text { manualmente }\end{array}$ \\
\hline
\end{tabular}

Fonte: Elaborado pelos autores, 2017.

\section{Registros Completos e Sua Importância}

Através dos registros se concretizava a formulação situacional da assistência efetivada, dos quais eram extraídas informações que perpassavam diagnósticos e características dos serviços, relacionando falhas e causas, e identificando a necessidade de práticas sistematizadas a fim de reestruturar a assistência.

Os registros eram visualizados como uma maneira prática de fonte de consulta sobre a condição de saúde dos pacientes e objeto de valorização profissional ${ }^{11}$.

Diante da ausência de registros detalhados dos atos, que por vezes não identificavam os horários da assistência ofertada e nem mesmo o profissional que Revista Inova Saúde, Criciúma, vol.9, n. 1, jul. 2019. 
executou a ação ${ }^{2}$, tal ausência se constituiu um entrave na execução do cuidado, onde a interação com instrumentos aplicáveis, diante de cada realidade, as anotações oportunizaram a confirmação de um cuidado integral e qualificado, e ainda foi um meio avaliativo do cuidado prestado a partir da adoção de indicadores de qualidade que mensuram tanto o processo como os resultados da assistência.

Em outro estudo ${ }^{15}$, foi constatado na observação realizada pelos enfermeiros, que não foi encontrado qualquer registro da equipe de enfermagem relacionado as condições da cavidade bucal dos pacientes, bem como, ausência de especificação para produtos utilizados para a realização do procedimento, mas com média de registros de efetividade do procedimento em $67,3 \%$, tais resultados podem ser interpretados como não executado ou não supervisionado, e o mais relevante como negligenciado, podendo ter consequências para os serviços e gestores.

Em um estudo ${ }^{1}$ realizado em uma Unidade de Terapia Intensiva no Mato Grosso do Sul, a avaliação da assistência foi classificada como limítrofe ou sofrível, levando-se em consideração os aspectos de estrutura e processo, utilizando como destaque para justificar tal resultado a ausência de profissionais em número suficiente, despreparados e com a carga de trabalho elevada, e ainda, identificou-se a necessidade de destinar tempo suficiente para a realização de avaliações críticas, e isto, independentemente da metodologia utilizada para o registro ${ }^{11}$.

Assim, o registro de todas as etapas da SAE, tem sua virtude no fato de atuar como facilitador da valorização da assistência de enfermagem, identificando os pontos fracos que necessitam de melhoria dentro do processo de trabalho².

\section{Solução Eletrônica?}

Diante dos achados nos estudos ${ }^{14,16}$, obteve-se dados que avaliavam diversas características nos registros eletrônicos de enfermagem e os registros realizados de forma manual. Em ambos os estudos, a conclusão era que positivamente um programa para anotações da equipe apresentavam vários tópicos que deixavam o processo de enfermagem mais complexo, porém conciso. De fato, em um estudo ${ }^{16}$, foi comprovado que o registro eletrônico se torna mais lento, no entanto, a forma informatizada de aplicabilidade, apresentava mais informações a serem extraídas, 
quando comparado aos registros manuais, pois este necessitava de capacitação do profissional durante sua prática gradativa da Sistematização da Assistência de Enfermagem, divergindo em alguns pontos de perspectiva entre profissionais.

Embora, haja a provisão de tempo superior durante os registros eletrônicos, foi percebida que a utilização de um software gerou várias possibilidades diagnósticas de prescrições de enfermagem, embora, em ambas as formas de registros permitam a aplicação correta do processo de enfermagem ${ }^{14}$, e que acabam por demarcar um cuidado mais estratificado e integral aos sujeitos.

Desta forma, embora o enfermeiro utilize mais tempo para estudar e selecionar os diagnósticos de enfermagem sugere-se que a realização desta tarefa seja mais confiável e precisa no registro eletrônico, no entanto, instrumentos impressos direcionados e de rápido preenchimento, podem representar também um importante processo de trabalho ${ }^{14}$, assim, foi evidenciado que tanto os formulários manuais como os eletrônicos, podem ser utilizados para a implementação do processo de enfermagem pois dispõem das funções necessárias a sua aplicação na forma correta, embora, houvesse encontrado a necessidade de atualização dos instrumentos impressos, onde estes encontravam-se desatualizados e com pouco espaço para a escrita, compelindo as anotações descritivas serem breves ${ }^{11}$.

Um software que abrange apenas o processo de enfermagem, faz com que o enfermeiro disponha de mais tempo para vivenciar as etapas do processo, habituando-se as práticas, proporcionando economia de tempo e evita registro redundantes ${ }^{14}$. O layout checklist foi identificado na maioria dos artigos como forma efetiva de registrar e avaliar a assistência, sendo uma estratégia amplamente abordada $^{1,2,14,16,17,18,19}$.

\section{CONCLUSÃO}

Diante da concisão do que foi encontrado na literatura, os autores citam a necessidade de registros completos e claros como forma de continuidade do cuidado, e fato este simples de ser executado e compreendido pelos profissionais. Como forma estratégica de melhoria da qualidade das anotações, impressos bem desenvolvidos e instrumentos eletrônicos para registros se demonstraram bem 
aceitos e com resolutividade adequada para problemas como: registros incompletos, escassos e redundantes, mas tendenciando a praticidade dos prontuários eletrônicos, como forma de aperfeiçoamento dos cuidados.

Apesar da vasta abordagem literária sobre a qualidade e importância de registrar os procedimentos realizados e a assistência prestada aos pacientes, este estudo possuiu a limitação pelo fato de haver poucos estudos relacionando tais temas às unidades de terapia intensiva, deixando um hiato sobre a qualidade assistencial especifica deste setor.

Assim, diante de um cenário com grande densidade tecnológica, como as unidades de terapia intensiva, sugere-se a necessidade de atualização contínua dos instrumentos de registros compatível com tal nível de tecnologia, atendendo a demanda de registros rápidos, porém completos e favorecendo consequentemente, ao tempo do ato de registrar dos profissionais e a qualidade da assistência.

\section{REFERÊNCIAS BIBLIOGRÁFICAS}

1. Silva RB, Loureiro MDR, Frota OP, Ortega FB, Ferraz CCB. Qualidade da assistência de enfermagem em unidade de terapia intensiva de um hospital escola. Rev. Gaúcha Enferm. [Internet]. 2013; 34 (4): 114-20. Disponível em: <http://seer.ufrgs.br/index.php/RevistaGauchadeEnfermagem/article/view/36792>. Acesso em: 15 abr. 2017

2. Silva JA, Grossi ACM, Haddad MCL, Marcon SS. Avaliação da qualidade das anotações de enfermagem em unidade semi-intensiva. Esc. Anna Nery [Internet]. 2012; $\quad 16 \quad$ (3): $\quad$ 576-82. $\quad$ Disponível em <http://www.scielo.br/scielo.php?script=sci_arttext\&pid=S1414-

81452012000300021>. Acesso em: 16 abr. 2017

3. Ochoa-Vigo K, Pace AE, Santos $C B$. Análise retrospectiva dos registros de enfermagem em uma unidade especializada. Rev. Latino-Am. Enfermagem [Internet]. 2003; $11 \quad$ (2): 184-91. Disponível em: 
<http://www.scielo.br/scielo.php?script=sci_arttext\&pid=S0104-

11692003000200007\&Ing=en\&nrm=iso >. Acesso em: 15 abr. 2017

4. Vituri DW, Matsuda LM. Os registros de enfermagem como indicadores da qualidade do cuidado: um estudo documental, descritivo exploratório e retrospectivo. Online Braz J Nurs [Internet]. 2008; 7(1). Disponível em: <http://www.objnursing.uff.br/index.php/nursing/rt/printerFriendly/j.16764285.2008.1241/295>. Acesso em: 18 abr. 2017

5. Pinto KA, Melo CMM. A prática da enfermeira em auditoria em saúde. Rev. esc. enferm. USP [Internet]. 2010; 44(3): 671-8. Disponível em <http://www.scielo.br/pdf/reeusp/v44n3/17.pdf >. Acesso em: 15 abr. 2017

6. Dias TCL, Santos JLG, Cordenuzzi OCP, Prochnow AG. Auditoria em enfermagem: revisão sistemática da literatura. Rev. bras. enferm. [Internet]. 2011;64(5): 931-37. Disponível em: <http://www.scielo.br/scielo.php?script=sci_arttext\&pid=S0034$71672011000500020 \&$ Ing=en\&nrm=iso>. Acesso em: 02 abr. 2017.

7. Paiva SMA. Qualidade da Assistência hospitalar: avaliação da satisfação dos usuários durante seu período de internação (Tese). São Paulo: Escola de Enfermagem de Ribeirão Preto da Universidade de São Paulo; 2006. Disponível em<http://www.teses.usp.br/teses/disponiveis/83/83131/tde-13122006-145412/>. Acesso em 29 abr. 2017.

8. Barreto JA, Lima GG, Xavier CF. Inconsistências das anotações de enfermagem no processo de auditoria. R. Enferm. Cent. O. Min.2016; 1(6): 2081-93. Disponível em: <http://www.seer.ufsj.edu.br/index.php/recom/article/view/917 >. Acesso em: 02 abr. 2017.

9. Campos GS. Registros de enfermagem: sua importância para a equipe e para o paciente. Escola GHC Instituto Federal de Educação, Ciência e Tecnologia do Rio 
Grande do Sul, Porto Alegre. 2016; (s/d). Disponível em: < http://colecionasus.bvs.br/lildbi/docsonline/get.php?id=904 >. Acesso em: 04 abr. 2017.

10. Padilha EF, Haddad MCFL, Matsuda LM. Qualidade dos registros de enfermagem em terapia intensiva: avaliação por meio da auditoria retrospectiva. Cogitare Enferm. 2014; 19(2): 239-45. Disponível em: <http://revistas.ufpr.br/cogitare/article/view/32103>. Acesso em: 15 abr. 2017.

11. Colaço A, Menezes FR, Nascimento ERP, Lazzarri DD, Böes AA, Jung W. Registro da avaliação de enfermagem em terapia intensiva: Discurso do sujeito coletivo. Rev Enferm da UFSM. 2015; 5 (2); 257-66. Disponível em: <https://periodicos.ufsm.br/reufsm/article/view/15509 >. Acesso em: 09 abr. 2017.

12. Chicharo SCR, Florêncio MV, Alves SZSP, Cortez EA, Andrade M, Valente GSC. Fatores facilitadores do ensino-aprendizagem na formação do enfermeiro: uma revisão integrativa. J. Res. Fundam. Care. Online. 2016; 8(2): 4099-108. Disponível em: $\quad$ <http://www.seer.unirio.br/index.php/cuidadofundamental/article/view/3408>. Acesso em: 04 abr. 2017.

13. Mendes KDS, Silveira RCCP, Galvão CM. Revisão integrativa: método de pesquisa para a incorporação de evidências na saúde e na enfermagem. Texto contexto - enferm. [Internet]. 2008; 17(4): 758-64. Disponível em: $<$ http://www.scielo.br/scielo.php?script=sci_arttext\&pid=S0104-

07072008000400018>. Acesso em: 04 abr. 2017.

14. Tannure MC, Lima APS, Oliveira CR, Lima SV, Chianca TCM. Processo de enfermagem: Comparação do registro manual versus eletrônico. J. Health Inform. 2015; 7(3): 69-74. Disponível em: <http://www.seer.ufsj.edu.br/index.php/recom/article/view/773>. Acesso em: 16 abr. 2017. 
15. Zanei SSV, Kawamura MM, Mori S, Cohrs CR, Whitaker IY. Valoração e registros sobre higiene oral de pacientes intubados nas unidades de terapia intensiva. Rev Min Enferm. 2016; 20 (s/n). Disponível em: <http://www.reme.org.br/exportar-pdf/1101/e965.pdf>. Acesso em: 18 abr. 2017.

16. Silva VCG, Betta CA, Nishio EA, Barsotini CNG, Wainer J. Mensuração do tempo dos registros manual e eletrônico da Sistematização da assistência de enfermagem em unidade de terapia intensiva. J. Health Inform. 2012; 4(2); 37-42. Disponível em: <http://www.jhi-sbis.saude.ws/ojs-jhi/index.php/jhi-sbis/article/view/173>. Acesso em: 16 abr. 2017.

17. Chaves NMO, Moraes CL.Controle de infecção em cateterismo vesical de demora em unidade de terapia intensiva. R. Enferm. Cent. O. Min. 2015; 5 (2):16507. Disponível em: <http://www.seer.ufsj.edu.br/index.php/recom/article/view/773>. Acesso em: 04 abr. 2017.

18. Barbosa TP, Beccaria LM, Poletti NAA. Avaliação do risco de úlcera por pressão em UTI e assistência preventiva de enfermagem. Rev Enferm UERJ. 2014; 22 (3): 353-8. Disponível em: $<$ http://www.epublicacoes.uerj.br/index.php/enfermagemuerj/article/view/13724>. Acesso em: 02 abr. 2017.

19. Menegueti MG, Martins MA, Canini SRMS, Basile-Filho A, Laus AM. Infecção urinaria em unidade de terapia intensiva: um indicador de processo para prevenção. Rev. RENE. 2012; 13(3): 632-8. Disponível em: <http://www.periodicos.ufc.br/index.php/rene/article/view/3998>. Acesso em: 09 abr. 2017 